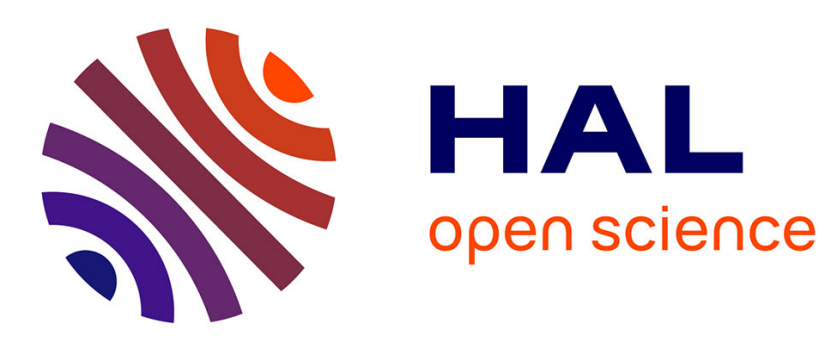

\title{
Facteurs institutionnels de la diversité des carrières scolaires
}

\author{
Marie Duru-Bellat, Alain Mingat
}

\section{To cite this version:}

Marie Duru-Bellat, Alain Mingat. Facteurs institutionnels de la diversité des carrières scolaires. Revue française de sociologie, 1987, 28 (1). hal-03201605

\section{HAL Id: hal-03201605 \\ https://hal.science/hal-03201605}

Submitted on 4 Jun 2021

HAL is a multi-disciplinary open access archive for the deposit and dissemination of scientific research documents, whether they are published or not. The documents may come from teaching and research institutions in France or abroad, or from public or private research centers.
L'archive ouverte pluridisciplinaire HAL, est destinée au dépôt et à la diffusion de documents scientifiques de niveau recherche, publiés ou non, émanant des établissements d'enseignement et de recherche français ou étrangers, des laboratoires publics ou privés.

\section{(이) $\$$}

Distributed under a Creative Commons Attribution - NonCommercial - NoDerivatives| 4.0 


\title{
Facteurs institutionnels de la diversité des carrières scolaires
}

\begin{abstract}
RESTMi
L’article vise à apporter des résultats empiriques, obtenus à l'aide d'une méthodologie rigoureuse, dans le débat central en sociologie de l'éducation, concernant la génération des inégalités sociales dans les carrieres scolaires des élèves. Il sattache notamment à quantifier le poids respectif des inégalités de réussite dans le système scolaire - telles quelles se génèrent petit à petil au long de la scolarité -- et des inégalités comportementales et institutionnelles -- qui se forment dans la période très courte des moments d'orientation. On montre que les inégalités de réussite nont qu'un impact limité pour expliquer la diversité sociale des carrières scolaires alors que c'est dans les moments dorientation que se génére l'essentiel des différences. En centrant lanalyse sur ces moments dorientation, on observe que les procedures ont tendance à entériner les demandes familiales, elles-mêmes socialement biaisées. Toutefois, la prise en comple de la demande nexplique quine part minoritaire des différenciations sociales propres aux procédures dorientation. On montre alors que les biais sociaux spécifiques à la procédure viennent majoritairement de pratiques différenciées des collèges non sans rapport avec les caractéristiques sociologiques de leur public.
\end{abstract}

Pour qui s’intéresse aux carrières scolaires des élèves, certains faits sont clairs et bien établis : d'une part, une grande diversité entre les individus (certains s'arrêtent lorsqu'ils en ont la possibilité alors que d'autres semblent vouloir toujours repousser leur sortie du système scolaire), d'autre part, des inégalités sociales marquées et relativement stables dans le temps. Un courant important de la sociologie de léducation analyse cette diversité en référence aux structures et au fonctionnement de la société. Un autre courant s’intéresse plus aux mécanismes concrets qui produisent ces résultats. Dans ce dernier cas de recherche, certains mettent davantage laccent sur les conditions et les facteurs associés aux acquisitions scolaires (sociologie du langage, sociologie des interactions dans la classe, sociologie de l'évaluation...), alors que d'autres, moins nombreux, s'attachent plutôt à l'analyse des mécanismes de l'orientation et de la sélection.

Ces deux dernières approches, dont l'intérêt spécifique est incontestable, méritent néanmoins d'être examinées de concert, dans le but d'avoir 


\section{Revue française de sociologie}

une version plus globale des mécanismes structurant la diversité des scolarisations et les biais sociaux associés. Ce n'est qu'en opérant ainsi qu'il est possible de distinguer les rôles respectifs des différences de réussite d'une part, des différences de comportement (des élèves, des familles, de l'institution scolaire...) d'autre part (1).

C'est dans cette perspective qu'a été structurée la recherche dont le présent article propose certains résultats (2). Il comprend deux parties :

- La première vise à séparer au niveau du palier d'orientation de fin de $5^{\text {ème }}$ les inégalités qui se sont sédimentées au cours des sept premières années de scolarité (il s'agit donc d'inégalités de réussite scolaire) et celles qui se nouent dans la phase d'orientation elle-même (inégalités de comportement, au sens large), à ce premier point de "bifurcation " dans le système scolaire. On montrera que les inégalités de réussite n'ont qu'un impact limité pour expliquer la diversité sociale des carrières scolaires (accès à la $4^{\mathrm{eme}}$ ), alors que c'est dans le moment de l'orientation que se génère l'essentiel des différences.

- Compte tenu de ce résultat empirique et en raison du caractère décentralisé de la procédure d'orientation (les décisions sont prises au sein de chacun des 30000 conseils de classe du système scolaire français), la deuxième partie de ce texte s'interroge sur une éventuelle diversité des pratiques d'un collège à l'autre. On montre alors que sont à l'œuvre des pratiques très différenciées et que celles-ci participent significativement à la génération des inégalités sociales propres à la procédure d'orientation.

Pour étudier la diversité des pratiques des collèges, deux approches pouvaient être envisagées. L'une consiste à analyser de l'intérieur les pratiques concrètes (approche de type ethnographique ou participante), l'autre à observer, de l'extérieur, les caractéristiques des acteurs (élèves, familles, professeurs, conseils de classe...) et à les confronter avec les résultats de l'action d'orientation, en cherchant à révéler (par des méthodes statistiques appropriées) les critères objectivement mis en œuvre. C'est cette dernière approche qui a été ici adoptée.

(1) Un premier essai d'analyse empirique de ces deux composantes des inégalités sociales quant à l'accès à un niveau donné d'éducation a été réalisé à propos de l'entrée en $6^{\mathrm{cme}}$ par A. Girard, H. Bastide, G. Pourcher dans "Enquête nationale sur l'entrée en $6^{\text {eme }}$ et la démocratisation de l'enseignement ", Population, 18, 1963, pp. 1-48, reproduit dans Population et l'enseignement (INED) Paris, Puf, 1970. L'intérêt théorique de ce type d'approche a été souligné par R. Boudon dans L'inégalité des chances. La mobilité sociale dans les sociétés industrielles, Paris, Colin, 1973.
(2) Il s'agit d'une enquête longitudinale portant sur 2500 élèves scolarisés en classe de $5^{\text {eme }}$ dans l'Académie de Dijon en 1982-83. Des informations très nombreuses ont été collectées concernant notamment, outre le déroulement précis de l'orientation, l'ensemble de la scolarité antérieure et la scolarité consécutive à l'orientation (cette phase de la recherche est actuellement en cours). M. Duru, A. Mingat, De l'orientation en fin de cinquieme au fonctionnement du college. 1. Evaluation de la procédure. Cahier de l'IREDU $\mathrm{n}^{\circ} 42$, Dijon, 1985. 


\section{1. - Sept années de scolarité, trois mois pour l'orientation. Quelle part dans la génération des inégalités sociales?}

\section{TABleAd I. - Taux de passage (\%) entre la classe de $5^{\text {ime }}$ et de $4^{\text {ime }}$ en fonction de quelques variables descriptives}

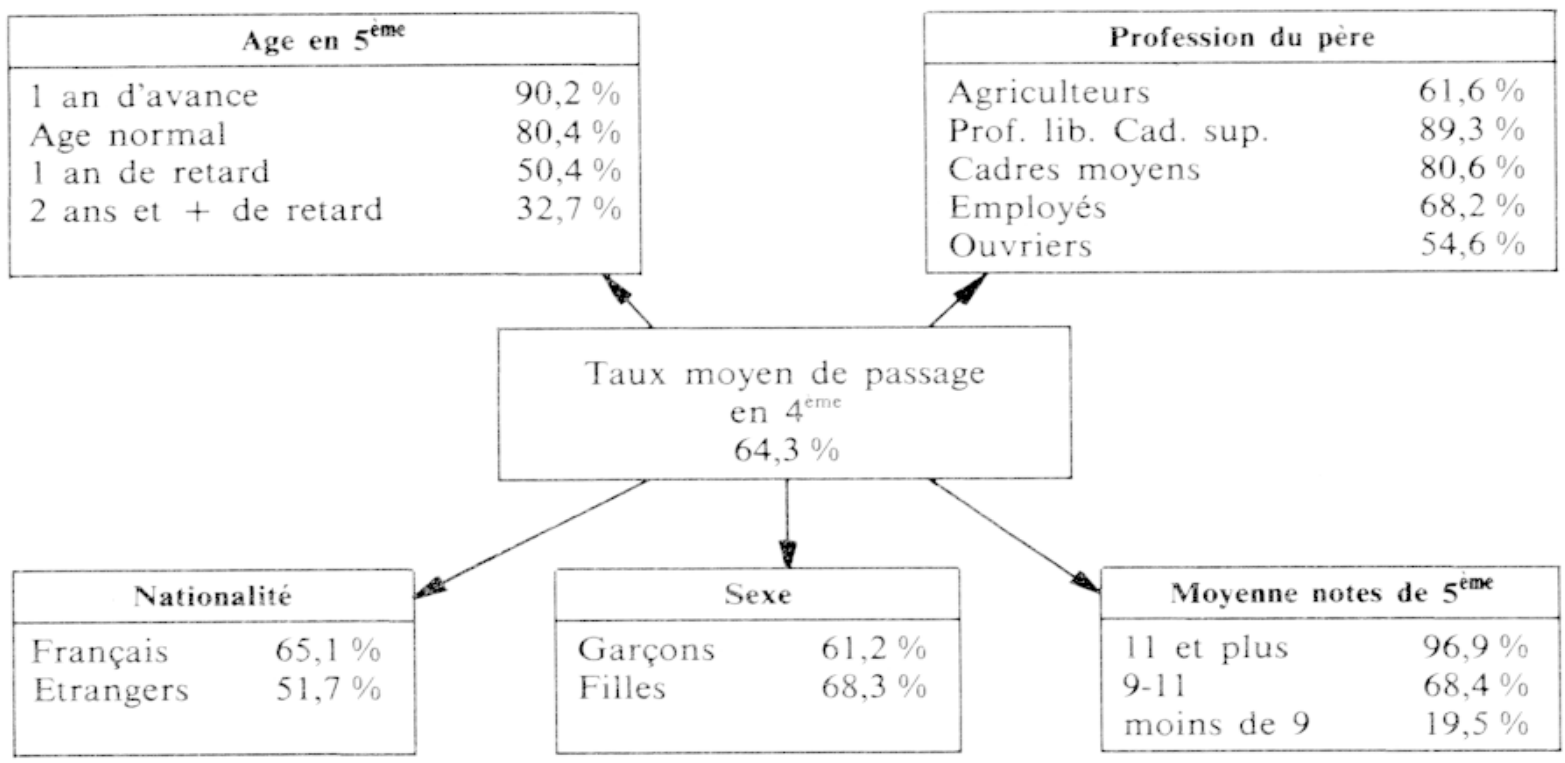

Au-delà du chiffre moyen du taux de passage de la $5^{\mathrm{emc}}$ à la $4^{\mathrm{cmc}}(64,3 \%)$ - 1/3 d'éliminés - ce sont les différences entre groupes qui sont surtout intéressantes. Ainsi, on observe des écarts substantiels entre les élèves selon leurs résultats scolaires ou selon leur origine sociale. On note par exemple qu'il y a près de 35 points d'écart entre le taux de passage des enfants d'ouvriers et celui des enfants de cadres supérieurs et professions libérales, à l'avantage de ces derniers.

Pour intéressants que soient ces chiffres, qui expriment les clivages réels à la fin de la procédure d'orientation, ils ne nous éclairent pas directement sur les mécanismes qui les ont engendrés. Pour aller dans cette direction, il est important d'une part de démêler le rôle respectif des différentes variables dont l'interaction produit le résultat observé et, d'autre part, de séparer les différences concrétisées avant la période d'orientation (tout au long de la carrière de l'élève) de celles qui se génèrent dans le temps de la procédure institutionnelle d'orientation.

Par exemple, pour ce qui est des interactions, on observe que les élèves plus âgés ont en général des notes inférieures, si bien qu'on ne peut affirmer d'emblée que le tableau précédent traduise correctement les effets de ces deux variables. De même, en ce qui concerne la séparation temporelle recherchée, il est clair que la catégorie sociale d'origine peut à la fois avoir eu un effet sur les notes obtenues ou l'âge en $5^{\text {ème }}$ (qui 
résultent en partie des différenciations scolaires antérieures) et exercer un impact net lors de la procédure d'orientation elle-même.

Pour opérer cette séparation analytique, il est nécessaire d'avoir recours à une modélisation des phénomènes étudiés. Nous avons retenu la forme générale suivante :

- Notes en $5^{\text {eme }}=\mathrm{f}$ (profession du père, sexe, nationalité...)

- Age en $5^{\mathrm{cme}} \quad=\mathrm{g}$ (profession du père, sexe, nationalité...)

- Orientation des enfants

en fin de $5^{\text {eme }}=\mathrm{h}$ (profession du père, sexe, nationalité..., notes en $5^{\text {ème }}$, âge en $5^{\text {eme }}$ )

Chacune des équations mesure l'impact de chacune des variables, "toutes choses égales par ailleurs" (celles introduites dans le modèle), alors que l'ensemble de la modélisation autorise d'une part à examiner l'impact de l'origine sociale sur les notes et l'âge en $5^{\text {cme }}$ et d'autre part à évaluer, au-delà des différenciations scolaires, les différenciations sociales spécifiques au moment de l'orientation. Il s'agit de modèles statistiques de régression. Ceux-ci sont linéaires pour l'estimation des deux premiers modèles, et logistiques (estimés par le maximum de vraisemblance) pour le troisième modèle, en raison du caractère probabiliste de la variable caractérisant l'orientation.

Le tableau II donne les résultats des estimations de la probabilité d'avoir accès à la classe de $4^{\text {eme }}$ (SR4) en fonction des origines sociales seules d'une part, des origines sociales et des résultats scolaires (notes, âge) d'autre part.

La première partie (A) du tableau II donne les écarts bruts entre catégories socio-professionnelles (c'est l'image directe, dans une structure de modèle, des différences dans les probabilités d'accès à la $4^{\text {ème }}$ telles que l'exprime le tableau I), alors que la seconde partie du tableau (B) modélise l'accès à la $4^{\text {eme }}$ en tenant compte des origines sociales, des notes obtenues et de l'âge. Les différences observées dans ce second tableau mesurent par exemple les écarts nets entre origines sociales à valeur scolaire et âge donnés. Dans l'hypothèse où la procédure d'orientation en elle-même fonctionnerait en moyenne de manière "méritocratique ", c'est-à-dire si les décisions finales d'orientation se prenaient sur la base de la valeur scolaire des élèves sans ajouter de biais sociaux systématiques, alors les coefficients attachés aux différentes modalités de la variable catégorie sociale devraient tendre vers zéro. Les différences sociales après l'orientation tiendraient alors uniquement au fait que les enfants issus des différents milieux se présentent à ce bilan qui "fonde » l'orientation avec des performances inégales (3).

(3) Cela bien sûr ne voudrait pas dire nécessairement que l'ensemble du système fonctionne de façon "méritocratique " puis- que ces différences traduiraient des inégalités sociales dans la réussite scolaire jusqu'au moment de lorientation. 
TABLEAL II. - Modèles expliquant les probabilités d'accès à la $4^{e m e}$ (SR4)

\begin{tabular}{|c|c|c|c|}
\hline \multicolumn{2}{|c|}{ Variables } & SR4 (A) & SR4 \\
\hline Référence & Active & $b$ & $b$ \\
\hline \multirow{7}{*}{ Ouvrier } & Agriculteur & $0.273^{* *}$ & 0.255 \\
\hline & P.I.C. & $0.253^{* * *}$ & 0.292 \\
\hline & Prof. lib.-Cad. sup. & $1,926^{* * *}$ & $1.428 * *$ \\
\hline & Cadres moyens & $1,226^{* * *}$ & $0,941^{* * *}$ \\
\hline & Employés & $0.566^{* * *}$ & $0.714^{* * *}$ \\
\hline & Sans profession & $-0.415^{* * *}$ & -0.076 \\
\hline & Sans père & 0,219 & $0.738^{* * *}$ \\
\hline \multicolumn{2}{|c|}{ Moyenne en $5^{\text {eme }}$} & & $0,956^{* * *}$ \\
\hline \multicolumn{2}{|c|}{ Age en $5^{\text {eme }}$} & & $-0,440^{* * *}$ \\
\hline \multicolumn{2}{|c|}{$\%$ de variance expliquée } & 0,218 & 0.705 \\
\hline
\end{tabular}

Légende: Les chiffres contenus dans ce tableau sont les coefficients b attachés aux différentes variables explicatives de la probabilité de passage dans une forme fonctionnelle logistique (SR4 $=1 / 1+\mathrm{e}^{-\left(h_{1}\right)+h_{1} x_{1}-1}$ ). Ils expriment limpact quantitatif des différentes variables, toutes choses égales par ailleurs. Ils permettent de calculer des effets marginaux sur la variable a expliquer selon l'expression suivante : $\Delta S R 4=b, \Delta X_{1}(S R 4)(1-S R 4)$. (Par exemple. 1 point en plus de moyenne en $5^{\text {eme }}$ accroît de $0.956 \times 0.643(1-0.643)$ soit $21.9 \%$ la probabilité davoir accès a la classe de $4^{\mathrm{emc}}$. au point moyen.) Les astérisques expriment le caractère plus ou moins significatif des coefficents estimés ( ${ }^{*}$ significatif au seuil de $10 \%:{ }^{* *}$ au seuil de $5 \% ; * * *$ au seuil de $1 \%$ ).

Ce n'est pas ce qu'on observe puisque, dans le second modèle, les coefficients de plusieurs catégories socio-professionnelles (professions libérales-cadres supérieurs, cadres moyens, employés et " sans père ") sont significativement différents de zéro. Leurs valeurs numériques s'ordonnent en allant des enfants d'ouvriers à ceux de cadres supérieurs. Le graphique ci-après permet de visualiser les principales lignes de force structurant le phénomène étudié.

\section{Deux principales observations peuvent être faites.}

La première concerne la forme du "faisceau » de courbes. Ainsi, chez les élèves ayant une moyenne supérieure ou égale à 12, la différenciation tant en ce qui concerne l'âge que l'origine sociale est faible puisqu'en toute hypothèse le taux de passage est très élevé. Par contre, chez les élèves moyens et faibles, l'âge et l'origine sociale modulent fortement les chances d'accès à la $4^{\text {eme }}$. Ainsi, pour les élèves ayant 10 de moyenne, la probabilité d'accès à la $4^{\text {eme }}$ est de $90 \%$ chez les enfants de cadres supérieurs, àgés de 12 ans, et seulement de $68 \%$ chez les enfants d'ouvriers du même âge. Chez les élèves de 14 ans, les probabilités correspondantes sont $79 \%$ et $47 \%$ respectivement. 
Revue française de sociologie

La seconde concerne le caractère disjoint des trois courbes caractérisant les enfants de cadres supérieurs de celles concernant les enfants d'ouvriers. Ainsi, à note donnée, un enfant de cadre supérieur a, quel que soit son âge et fût-il très âgé, des probabilités d'accès en $4^{\text {ème }}$ plus fortes que celles d'un enfant d'ouvrier fût-il très jeune. Par exemple, en se reportant à la population des élèves ayant 10 de moyenne, un enfant de cadre ayant deux ans de retard a une probabilité de $79 \%$ contre $68 \%$ pour un enfant d'ouvrier n'ayant jamais redoublé. Une autre lecture du graphique peut être faite en lisant les probabilités de "façon horizontale ", avec l'observation qu'un enfant de cadre supérieur ayant un an de retard et 10 de moyenne a une probabilité d'accès à la $4^{\text {ème }}$ comparable à celle d'un enfant d'ouvrier de même âge avec 11,7 de moyenne. Il faudra donc 1,7 point de moyenne en plus pour compenser le poids de l'origine sociale.

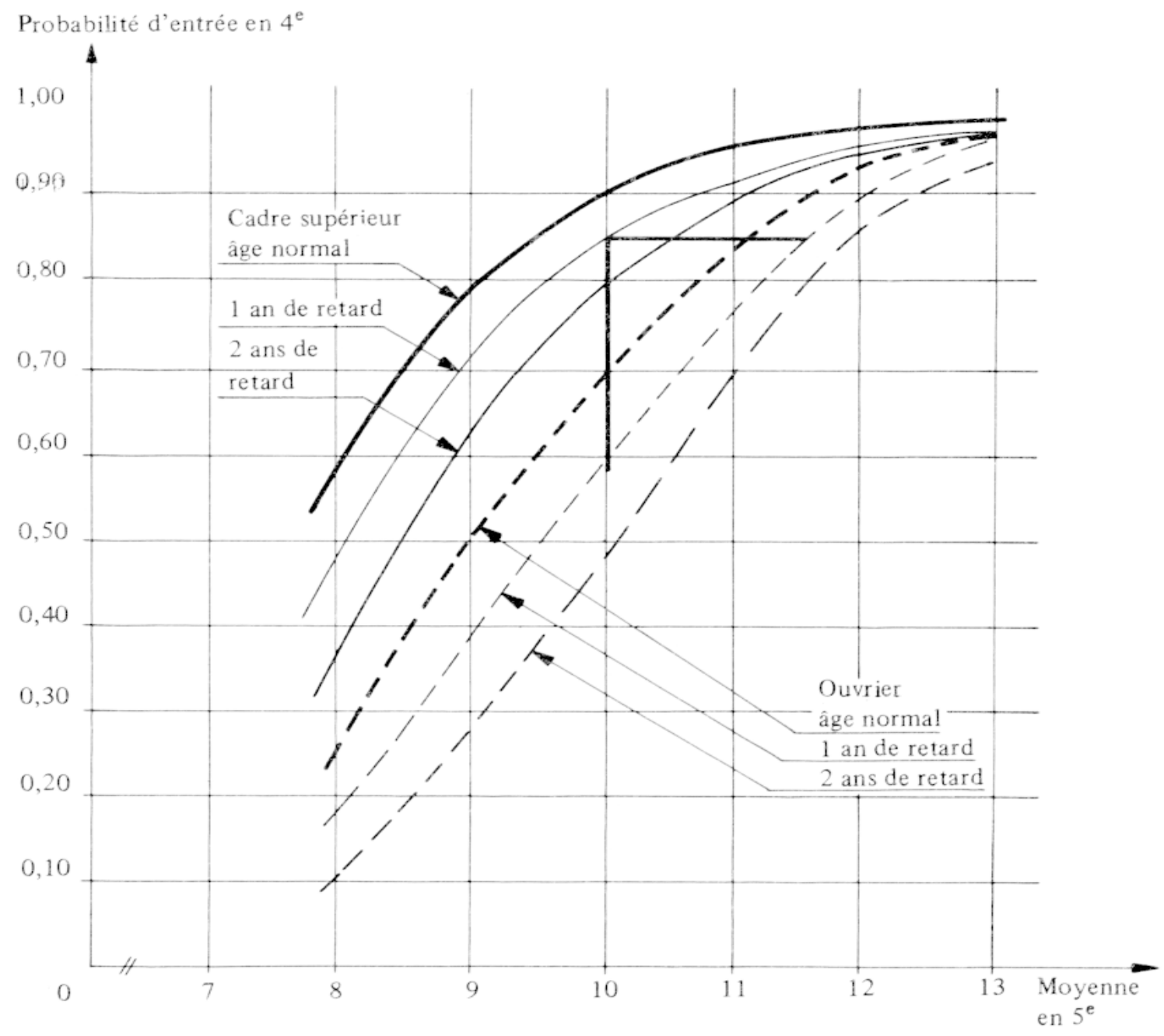

La procédure modélisée permet de décomposer les écarts bruts d'accès à la $4^{\text {ème }}$ entre les différentes origines sociales (telles que les exprimait le tableau I), en distinguant, d'une part, un écart tenant au fait que les enfants 
des différents milieux n'arrivent pas en $5^{\mathrm{eme}}$ avec les mêmes acquis scolaires ni au même âge et, d'autre part, un écart traduisant la dimension sociale du fonctionnement de l'orientation pour des élèves de valeur scolaire donnée. Cette distinction opère une séparation temporelle entre les inégalités sociales accumulées petit à petit dans les acquisitions scolaires des élèves tout au long de leur scolarité et celles qui se génèrent transversalement dans le moment de l'orientation. Par exemple, les écarts bruts qui séparent les enfants d'ouvriers des enfants de cadres quant au passage en $4^{\text {eme }}$ est de $34,7 \%(89,3-54,6)$ pour les cadres supérieurs et $26 \%(80,6$ - 54,6) pour les cadres moyens; les enfants de cadres arrivent en $5^{\mathrm{eme}}$ avec, en moyenne, de meilleurs résultats scolaires à des âges plus jeunes; l'impact de ces différences de valeur scolaire entre enfants de cadres et d'ouvriers peut être chiffré à partir des estimations économétriques : ils sont de $9,0 \%$ pour les cadres supérieurs et de $6,0 \%$ pour les cadres moyens. Il résulte donc que $25,7 \%(34,7-9,0)$ est une mesure de l'écart des taux de passage entre enfants de cadres supérieurs et d'ouvriers, qui ne trouve pas ses racines dans les inégalités de valeur scolaire mais se génère au cours de la procédure d'orientation. Pour les cadres moyens, le chiffre correspondant est de 20,0\% $(26,0-6,0)$.

Il apparaît donc clairement que les inégalités sociales observées à l'issue du passage $5^{\mathrm{emc}} / 4^{\mathrm{eme}}$ se "produisent " majoritairement dans le moment de la procédure, alors que celles qui se sont constituées auparavant, et notamment au cours des sept années de scolarité, n'apportent qu'une contribution minoritaire. Les chiffres ci-dessus permettent d'estimer que seulement $26 \%(9,0 / 34,7)$ de l'écart brut initial entre taux de passage des enfants d'ouvriers et de cadres supérieurs (et $23 \%$ pour les cadres moyens) est dû aux différences de réussite scolaire, alors que $74 \%$ (77\% pour les cadres moyens) se nouent au cours de l'orientation elle-même.

Ces chiffres, bien sûr, doivent être considérés comme des ordres de grandeur, notamment en raison d'imprécision dans les mesures, des contraintes inhérentes à la modélisation (bien que les parts de variance expliquée soient élevées) et enfin du caractère "grossier " de la mesure des carrières scolaires par le seul fait de passer ou non en $4^{\text {eme }}$, indépendamment des variétés des acquisitions scolaires de ceux qui passent. Il n'en reste pas moins que ces réserves n'affectent pas l'ordre de grandeur des effets qui ont été ici distingués, à savoir que la variété sociale des carrières scolaires n'a pas principalement pour origine les inégalités sociales d'acquisition et de réussite à l'école (4).

Ce résultat, en creux, manifeste à l'évidence l'importance du moment de l'orientation. Sur le plan pratique, l'orientation en fin de $5^{\text {eme }}$ prend la

(4) Ce résultat a été obtenu à partir de l'opposition entre passage et non-passage en $4^{\text {eme }}$. Il est clair qu'au sein de la population admise en $4^{\text {eme }}$, il subsiste de notables différenciations scolaires qui se manifesteront dans la scolarité ultérieure. Toutefois, il faut aussi souligner que la partition opérée à ce stade esı décisive... principalement pour ceux qui ne passent pas en $4^{\text {eme }}$ (presque un enfant sur deux dans le milieu ouvrier). 
forme d'une interaction entre des demandes familiales d'une part (qui s'expriment en premier - intentions, vœux définitifs) et des conseils de classe (recommandations puis propositions définitives), suivie d'une affectation administrative des élèves dans les places disponibles avec la possibilité d'un appel de la part des familles.

Il est important de souligner que ce processus est suscité par les demandes familiales, les conseils de classe effectuant leurs propositions au vu de celles-ci et de la valeur scolaire des élèves. L'analyse de la demande d'une part et la mesure du poids de la demande dans les décisions finales d'autre part apparaissent a priori comme deux éléments clefs pour comprendre le fonctionnement de la procédure et sa place dans la génération des inégalités. Le schéma ci-dessous décrit les principales interactions entre variables.

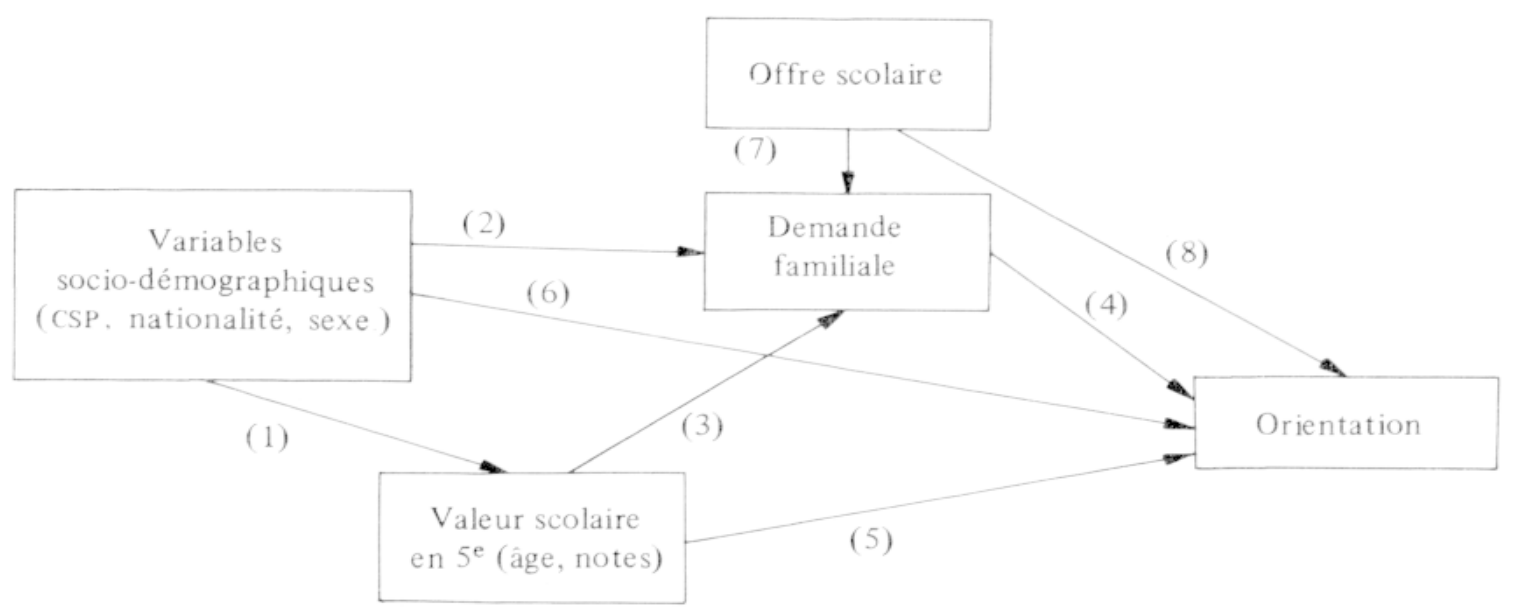

(1) mesure les différenciations «sociales» dans la valeur scolaire de l'élève en $5^{\text {eme }}$ (telle qu’elle résulte de sa scolarité antérieure).

(2) mesure les différenciations « sociales" (au sens large : catégorie socio-professionnelle de la famille, sexe, nationalité, taille de la famille...) dans la demande familiale à valeur scolaire de l'élève donnée.

(3) mesure l'impact de la valeur scolaire de l'élève sur la demande familiale, indépendamment des autres caractéristiques, notamment "sociales », de l'élève.

(4) mesure l'impact net de la demande sur les orientations finales des élèves, à valeur scolaire des élèves donnée et à demande familiale et offre scolaire données.

(5) mesure l'impact net de la valeur scolaire de l'élève sur son orientation, les autres facteurs étant donnés (aspect méritocratique de l'orientation).

(6) mesure l'effet «résiduel » des variables « sociales » qui ne transitent ni par la demande familiale, ni par la valeur scolaire de l'élève.

(7) et (8) mesurent l'effet net de l'offre scolaire sur les demandes familiales d'une part, les orientations effectives de l'élève d'autre part.

Chronologiquement, les variables démographiques et sociales exercent un impact sur la "production" de la valeur scolaire de l'élève, qui se concrétisera au moment de l'orientation par un âge à un certain niveau 
d'acquisition. Ensuite, la demande familiale (qui traduit les projets et les contraintes des familles) va s'exprimer, vraisemblablement, en fonction de la valeur scolaire de l'élève (auto-sélection scolaire) et aussi, on peut le penser, avec des différenciations sociales à valeur scolaire donnée (auto-sélection sociale). D'autres facteurs, tels que l'offre de places, peuvent également avoir une influence sur la demande. Enfin, le conseil de classe va élaborer ses propositions en "combinant " la valeur scolaire de l'élève, la demande exprimée et d'autres facteurs plus spécifiques, en incorporant éventuellement de nouveaux biais sociaux (positifs ou négatifs) à valeur scolaire et demande données.

Pour démêler ces différents phénomènes et évaluer leurs impacts respectifs, des modèles d'un type comparable à ceux présentés précédemment ont été estimés. Les principaux résultats de ces estimations sont les suivants.

1. La génération de la demande. On constate que les familles ont en moyenne d'autant plus tendance à demander la quatrième que leurs enfants sont jeunes et ont de bons résultats, mais cette auto-sélection des familles s'exerce avec plus ou moins d'intensité selon les milieux sociaux. En fait, seules les familles de cadres (moyens et supérieurs) se distinguent de l'ensemble des autres familles. Par exemple, pour des élèves moyens (en termes de résultats scolaires et d'âge), la probabilité de demander une quatrième est de $77 \%$ pour les enfants d'ouvriers, employés, agriculteurs, commerçants, alors qu'elle est de $95 \%$ pour les enfants de cadres supérieurs. Ceci vaut pour les élèves moyens. Pour les élèves brillants et jeunes, les biais sociaux sont pratiquement inexistants alors que les différenciations se creusent dès lors que les élèves sont plus âgés et/ou moins bons scolairement.

2. L'impact de la demande sur les orientations effectives. Ces phénomènes d’auto-sélection différenciée seraient d'un intérêt limité si la demande s'avérait exercer un impact faible sur la détermination de l'orientation effective des élèves. Le fonctionnement réel de l'orientation est tout autre. Si dans un premier temps on cherche des critères globaux de "pronostic " du sort de l'élève, on observe qu'il est à peu près aussi utile de connaître la demande familiale que le niveau scolaire de l'élève. Cela dit, ceci ne signifie pas que ces deux critères soient parfaitement substituables. En effet, les facteurs qui structurent les demandes familiales, bien que globalement les mêmes que ceux qui déterminent l'orientation effective, ne se combinent pas dans les mêmes proportions : ainsi, les familles accordent "spontanément " à l’âge un poids négatif significativement plus important que ce qui va s'avérer être le poids réel de l'àge in fine. Elles accordent par ailleurs un poids moindre aux résultats scolaires. Ces différences entre structuration de la demande et orientation effective témoignent de l'action exercée par les conseils de classe. Par exemple, en ce qui concerne l'âge, qui constitue bien réellement un facteur d'orientation, il convient de souligner que les conseils de classe tendent à limiter 
son impact par rapport au poids que lui affectent "spontanément " les familles.

On peut aussi, de façon analytiquement plus précise, estimer des modèles intégrant simultanément la valeur scolaire, la demande et l'origine sociale. Les estimations statistiques montrent qu'au-delà du rôle de la valeur scolaire (sur la demande et directement sur les orientations), la demande exerce bien un impact net attestant de la réceptivité du conseil de classe aux pressions de la demande familiale. Toutefois, pour important que soit l'effet net de la demande sur les orientations effectives des élèves, il reste des biais sociaux substantiels à valeur scolaire et demande données. Il est possible de chiffrer ces différentes composantes : ainsi, dans les inégalités sociales, quant au passage - ou non -.- en classe de $4^{\text {eme }}$, un quart des différenciations entre enfants de cadres - supérieurs ou moyens - d'une part, enfants d'ouvriers ou d'agriculteurs de l'autre, s'explique par des différences sociales nettes dans l'expression de la demande. C'est dire que l'expression de la demande, telle qu'elle se formule pendant les quelques mois de la phase d'orientation, engendre autant d'inégalités sociales que celles qui se sont constituées petit à petit dans une réussite scolaire inégale tout au long des sept années de scolarité antérieure.

En dépit de la force du résultat précédent, ces mêmes estimations statistiques expriment aussi qu'au-delà de l’impact des différences sociales de réussite scolaire de l'élève et de demande familiale sur les orientations, une part importante (environ la moitié) subsiste, sur laquelle il est pertinent de s'interroger.

Pour avancer dans cette direction, un fait mis en évidence par un modèle statistique est d'une importance particulière. En effet, en introduisant dans un même modèle les différents collèges (comme variables discrètes) en sus des variables prises en compte précédemment, on observe la disparition de l'effet des variables sociales. C'est dire qu'en moyenne, en portant l'observation au niveau du collège, on n'observe plus de biais sociaux spécifiques quant à l'accès à la quatrième, à valeur scolaire et à demande données. ('est dire aussi que l'essentiel des biais sociaux "résiduels" (environ la moitié des différenciations sociales globales d'accès à la $\left.4^{\mathrm{cme}}\right)$ provient du fait d'une part que les collèges sont assez typés socialement (ce que l'on sait) et d'autre part que les colleges ont des pratiques en matière d'orientation différentes et non sans rapport avec les caractéristiques sociologiques de leur public, qu'il convient maintenant d'étudier.

\section{II. - Une variété dans les pratiques des collèges}

Le modèle statistique duquel a été extrait le résultat précédent (à savoir qu'une partie appréciable des inégalités sociales transite par des différences inter-collèges) témoigne du même coup que les collèges ont des politiques significativement différentes en matière d'orientation puisque, 
à valeur scolaire, demande et origine sociale données, les coefficients attachés à la plupart des collèges sont significatifs. Par exemple, pour tel collège (non extrême) de notre échantillon, l'estimation statistique indique qu'on aurait pu s'attendre, vu les caractéristiques de son public, à ce que son taux de passage en $4^{\text {eme }}$ soit de $16 \%$ plus élevé (si ses pratiques d'orientation étaient celles de la moyenne des établissements de l'échantillon). Il est ici important de souligner que ces différences de sélectivité entre collèges s'appliquent de fait à l'ensemble des élèves d'un même collège; indépendamment de leur origine sociale, tous les élèves d'un méme collège vont bénéficier, ou pâtir, du climat plus ou moins sélectif qui y règne, sachant qu'on observe qu'en moyenne les collèges qui accueillent les publics les plus populaires ont tendance à être les plus sélectifs.

Il faut préciser que ces différences de sélectivité des collèges - à savoir pour un élève de caractéristiques scolaires et sociales données d'avoir des chances différentes de passer en $4^{\text {eme }}$ selon le collège où il est scolarisé ont été établies sur la base de la valeur scolaire des élèves mesurée par les notes. Or, les travaux de docimologie ne peuvent que nous inviter à la prudence quant à l'utilisation de ces notes pour des comparaisons inter-collèges. Pour remédier à ces difficultés, des épreuves de connaissances (en mathématiques et en français) ont été passées par tous les élèves de l'échantillon et corrigées de façon centralisée.

L'analyse des pratiques d'orientation des collèges reprise sur la base des épreuves propose une image relativement différente de celle donnée par la prise en compte des notes : les différences de sélectivité entre collèges, à âge et résultats aux épreuves communes donnés, sont réduites, mais les écarts restent néanmoins substantiels. Ainsi, en moyenne, on peut estimer que les chances d'accès à la $4^{\text {ème }}$ pour des élèves véritablement comparables sont affectés d'un aléa d'environ $10 \%$ selon le collège où ils sont scolarisés. C'est dire qu'un élève ayant par exemple une probabilité de $70 \%$ d'avoir accès à la $4^{\text {cme }}$ dans un collège moyen n'aurait que $60 \%$ de chance en moyenne dans le groupe des collèges sélectifs et $80 \%$ en moyenne dans le groupe des collèges "généreux " (5). Ces écarts restent donc considérables. Ils ont en outre une « légitimité " particulière puisque les élèves ont été évalués sur une base commune, indépendamment des pratiques locales de notation.

En dépit de l'opinion positive qu'on peut avoir sur l'utilisation des épreuves communes, il est néanmoins nécessaire de revenir aux notes scolaires dans la mesure où les modèles rendant compte de l'orientation à l'aide des notes ont un pouvoir explicatif plus élevé que celui des modèles fondés sur les épreuves communes (6), attestant par là-même que les conseils de classe ont tendance à valider, par leurs décisions, les

(5) Les écarts seraient ieaucoup plus larges si on prenait le cas des collèges plus extrêmes. Ainsi, la fourchette des extrêmes serait $(-32 \% ;+20 \%)$.

(6) Ainsi. le modèle orientation $4^{\mathrm{eme}}$ (no- tes, âge, sexe) explique près de $70 \%$ de la variance de l'orientation, alors que le chiffre correspondant n'est que de $51 \%$ si on substitue le résultat aux épreuves communes aux notes obtenues. 
jugements qu'ils ont antérieurement portés sur les élèves. La connaissance simultanée, dans cette enquête, des notes et résultats aux épreuves communes nous permet d'analyser plus finement le rôle des notes dans l'orientation en séparant ce qui relève des pratiques de notation d'une part, de leur prise en compte dans la décision d'orientation de l'autre.

Pour ce qui est des pratiques de notation, elles sont définies comme des biais systématiques (positifs ou négatifs) dans les notes données à des élèves de valeur scolaire donnée comparable (sur la base des épreuves communes). On observe des différences marquées entre collèges. Ainsi, un élève de valeur scolaire donnée verra en moyenne sa note de mathématiques varier de \pm 1 point selon qu'il est dans un collège en moyenne indulgent ou sévère; cet écart est d'environ $3 / 4$ de points en français. L'ampleur de ces différences a comme conséquence factuelle qu'il n'y a aucune relation statistique entre le niveau moyen des notes données dans un collège et le niveau moyen de la valeur scolaire (mesurée par les épreuves communes) dans ce même collège. Cette situation s'observe en dépit du fait qu'au niveau individuel la corrélation est substantielle entre notes et épreuves communes $(\rho=+0,65)$. C'est dire que, s'il y a bien cohérence dans le classement des élèves entre notes et épreuves communes à l'intérieur d'un collège, le niveau moyen des notes données dans le collège est sans rapport avec le niveau des élèves estimé dans une évaluation externe, manifestant ainsi des pratiques spécifiques et autonomes de notation (7).

Ces notes, diversement sévères, vont ensuite être utilisées, selon des modalités variées, pour produire, avec d'autres facteurs, une orientation plus ou moins sélective. On observe en effet qu'en moyenne tout se passe comme si les collèges avaient partiellement conscience du fait qu'ils sont sévères ou indulgents pour noter les élèves. En effet, plus un collège a tendance à noter sévèrement ses élèves, moins il aura tendance à être sélectif pour le passage en $4^{\mathrm{eme}}$ à notes données. Il y a donc en moyenne une compensation entre sévérité de la notation et sélectivité de l'orientation (8). Toutefois, cette compensation n'est que partielle puisqu'on observe qu'à travers elle les établissements ne réduisent que d'un quart l'impact que pourrait avoir la diversité de leurs notes sur l'orientation des élèves. On retrouve, par là-même, le constat fait précédemment, à savoir que la notation, telle qu'elle est pratiquée, a bien un impact et participe à l'explication de la diversité des pratiques d'orientation des collèges.

Les diversités des pratiques dont nous avons fait état jusqu'à présent concernent essentiellement le degré de sélectivité (et les facteurs qui la structurent), c'est-à-dire la définition du "combien d'élèves" sont autorisés à avoir accès à la quatrième. La diversité des collèges peut également

(7) Ce constat rappelle qu'à l'évidence la notation n`a pas pour seule fonction l'évaluation sommative des élèves.

(8) Ceci ne vaut qu'en moyenne car on observe des établissements à la fois sévères

dans leur notation et sélectifs dans l'orientation à notes données et des établissements indulgents dans leur notation et généreux en ce qui concerne le passage en $4^{\mathrm{cme}}$. 
s'exprimer par les modalités utilisées pour le choix des élèves orientés en $4^{\text {eme }}$. Ainsi, au-delà du degré de sélectivité qui définit le nombre d'élèves orientés en $4^{\text {ème }}$ (à quel niveau la barre est placée), la détermination individuelle des élèves retenus suppose l'utilisation de critères de classement entre élèves. On observe alors que, selon les collèges, les classements implicites des élèves se font sur une base diversifiée, c'est-à-dire qu'il y a des variétés dans le "comment les élèves sont choisis".

Cette variété revêt probablement des aspects multiples. Dans ce texte, nous étudierons la possibilité que, d'une part, les critères implicitement et effectivement pris en compte par les différents collèges ne soient pas identiques et, d'autre part, que l'interaction entre les familles et les conseils de classe se déroule selon des modalités différentes.

- Malgré l'intensité des lignes de force qui structurent globalement le processus d'orientation (présenté dans la première partie), on observe des différences systématiques dans les critères utilisés d'un collège à l'autre. Ces différences concernent le poids donné aux notes et l'importance respective accordée au français et aux mathématiques. Par exemple, alors que, dans l'ensemble de l'échantillon, français et mathématiques jouissent d'un poids comparable dans les décisions d'orientation, certains collèges accordent un poids sensiblement plus important à une matière plutôt qu'à une autre. Toutefois, le domaine où les différences entre collèges sont les plus massives concerne l'impact de l'âge de l'élève. Alors que l'âge apparaît globalement exercer un effet important (à valeur scolaire donnée) sur l'orientation, il convient de souligner que ce résultat est le produit d'un effet de composition entre des collèges qui lui donnent un poids très élevé et d'autres dans lesquels l'âge n'est pas pris, en lui-même, en considération. Ces derniers représentent près d'un collège sur deux. Ces variétés, dont les chiffres précédents témoignent de l'ampleur, vont constituer une source d'incohérence inter-individuelle dans la mesure où des élèves de profil donné seront appréciés différemment dans les jugements des conseils de classe. Ces phénomènes d'" incohérence " sont particulièrement lourds de conséquences pour les élèves moyens (9).

- L'interaction entre familles et conseils de classe a été étudiée dans cette recherche à partir du poids net que prend, in fine, la demande, dans les orientations effectives. En portant cette analyse au niveau du collège, on note que l'effet net de la demande est diversement marqué puisque dans certains collèges les décisions finales se fondent en totalité sur des critères scolaires, la demande des familles n'exerçant aucun impact net. Dans d'autres, par contre, la pression propre de la demande marque fortement les orientations finales au point qu'il est plus important de connaître la demande familiale que la valeur scolaire de l'élève pour rendre compte de son

(9) D’une façon générale, pour l'ensemble des résultats mis en évidence dans cette recherche, les effets observés valent particulièrement pour les élèves moyens. En effet, c'est sur cette population que les différen- ciations sociales, de sexe, d’âge ou de "traitement " entre collèges seront les plus manifestes. Chez les élèves très brillants, ou très faibles, ces différenciations n'auront qu'un effet limité. 
orientation. Dans la mesure où nous avons noté que la prise en compte des demandes familiales (socialement marquées) agissait comme une courroie de transmission des inégalités sociales, on comprendra que la diversité des pratiques des collèges dans la gestion de cette interaction soit un des aspects de la dimension inter-collèges des inégalités sociales dans les carrières scolaires.

Les analyses présentées dans cet article font apparaître un certain nombre de résultats factuels. Ils sont susceptibles d'être intégrés tant dans les débats théoriques en sociologie de l'éducation que dans ceux qui, ayant une vision plus opérationnelle, sont orientés vers la réflexion pour la politique de l'éducation. Ils appellent aussi des prolongements visant notamment à mieux cerner les mécanismes qui sous-tendent les relations mises en évidence. Par exemple, la variété des pratiques inter-collèges - qui interroge à la fois le sociologue, en ce qu'elle participe pour une part substantielle à la génération des inégalités sociales de carrière scolaire, et l'acteur de terrain et/ou le politique, en ce qu'elle manifeste des marges de manœuvre importantes - demande elle-même des investigations supplémentaires. En particulier, il conviendrait de rechercher les facteurs qui structurent cette diversité : rôle de l'offre locale de places, structure du corps enseignant, politique de constitution des classes... Une direction de recherche, connexe à cette dernière, pourrait consister à identifier, au sein de la variété des pratiques des collèges, celle qui serait la plus pertinente au plan de la qualité du pronostic que fait implicitement l'orientation quant à la carrière scolaire ultérieure de l'élève. Ces deux pistes de recherche complémentaires font actuellement l'objet de la suite de ce travail dont les premiers résultats sont résumés dans cet article.

Marie DURU, Alain MINGAT

IREDU-CNRS, Faculté des sciences Mirande, Université de Dijon, BP 138, 21000 Dijon 